\title{
Autotitrating versus standard noninvasive ventilation: a randomised crossover trial
}

\author{
J. Jaye*,\#, M. Chatwin*, M. Dayer*, M.J. Morrell*,\# and A.K. Simonds*,\#
}

ABSTRACT: The aim of the present study was to compare the efficacy of automatic titration of noninvasive ventilation (NIV) with conventional NIV in stable neuromuscular and chest wall disorder patients established on long-term ventilatory support.

In total, 20 neuromuscular and chest wall disease patients with nocturnal hypoventilation treated with long-term NIV completed a randomised crossover trial comparing two noninvasive pressure support ventilators: a standard bilevel ventilator (VPAP III) and a novel autotitrating bilevel ventilator (AutoVPAP). Baseline physiological measurements, overnight polysomnography and Holter monitoring were repeated at the end of each 1-month treatment period.

Nocturnal oxygenation was comparable between the autotitrating device and standard ventilator, as were sleep efficiency, arousals and heart rate variability. However, there was a small significant increase in mean overnight transcutaneous carbon dioxide tension (median (interquartile range) $7.2(6.7-7.7)$ versus $6.7(6.1-7.0) \mathrm{kPa}$ ) and a decrease in percentage stage 1 sleep (mean \pm SD $16 \pm 9$ versus $19 \pm 10 \%$ ) on autotitrating NIV compared with conventional NIV.

Autotitrating noninvasive ventilation using AutoVPAP produced comparable control of nocturnal oxygenation to standard nonivasive ventilation, without compromising sleep quality in stable neuromuscular and chest wall disease patients requiring long-term ventilatory support for nocturnal hypoventilation.

KEYWORDS: Automatic titration, chest wall disorders, neuromuscular disease, nocturnal hypoventilation, noninvasive ventilation

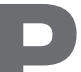

atients with neuromuscular or chest wall disease often develop restrictive ventilatory disorders as their disease progresses, resulting in hypercapnic respiratory failure. Nocturnal noninvasive ventilation (NIV) has been shown to increase survival [1-3], normalise nocturnal ventilation, and improve both daytime arterial blood gas control and sleep architecture $[4,5]$ in patients with chronic hypercapnic respiratory failure.

Establishing a patient on NIV requires specialist knowledge [6, 7]. Healthcare professionals must be adequately trained in selection of appropriate patients, choice of ventilatory mode, operation of the ventilator, mask fitting and adjusting NIV settings to optimise patient ventilation and comfort, and be able to support and educate patients on NIV use at home.

Automatic titration of continuous positive airway pressure (CPAP) in the treatment of obstructive sleep apnoea (OSA) is now common practice [8] and has been validated against conventional manual titration $[9,10]$. However, the algorithms and technology required to titrate NIV automatically have only recently been developed and as yet no validation studies have been published. Therefore, the aim of the present study was to evaluate the efficacy of automatically titrated NIV, compared with conventional manually titrated NIV, in patients with restrictive ventilatory disorders, specifically those with congenital and acquired neuromuscular disease or chest wall disease. All patients were experienced NIV users prior to the current study but were naïve to both ventilators used. The present authors tested the hypothesis that variable positive airway pressure ventilation (VPAP) delivered by a VPAP ventilator with pressure-support ventilation (PSV) titrated automatically could ventilate a patient as effectively as a standard VPAP ventilator with settings determined clinically by a skilled healthcare professional.

\section{MATERIALS AND METHODS}

Participants were recruited from the Royal Brompton Hospital Ventilator Clinic (London,
AFFILIATIONS

*Sleep and Ventilation Unit, Royal Brompton Hospital, and

${ }^{\#}$ Clinical and Academic Unit of Sleep and Breathing, National Heart and Lung Institute, London, UK.

CORRESPONDENCE

J. Jaye

Sleep and Ventilation Unit

Royal Brompton Hospital

Sydney Street

London

SW3 6NP

UK

Fax: 442073518911

E-mail: j.jaye@rbht.nhs.uk

Received:

April 282008

Accepted after revision:

September 132008

SUPPORT STATEMENT

Clinical Trials Registration No

NCT00252252, available at

www.clinicaltrials.gov

M.J. Morrell is funded by the

Wellcome Trust (London, UK)

STATEMENT OF INTEREST

Statements of interest for J. Jaye,

M.J. Morrell and A.K. Simonds, and for the study itself, can be found at www.erj.ersjournals.com/misc/

statements.shtml 
UK). In total, 41 patients fulfilled the inclusion criteria. Of these patients, 25 were recruited and 20 patients successfully completed the protocol (fig. 1). Inclusion criteria were: being $>18$ yrs old with congenital or acquired neuromuscular disease or chest wall disease and nocturnal hypoventilation, defined as nocturnal peak transcutaneous carbon dioxide tension $\left(P_{\mathrm{tc}}, \mathrm{CO}_{2}\right)>6.5 \mathrm{kPa}$. All patients were experienced ventilator users on long-term nocturnal NIV for a minimum of 6 months, and all had been stable and free from respiratory tract infection/acute exacerbation for $\geqslant 3$ months.

Patients were excluded if they had previous experience using a VPAP device (VPAP ${ }_{\circledR}$ models; ResMed, Bella Vista, Australia), to ensure that they were unfamiliar with the ventilators being compared, or if they had uncontrolled respiratory failure (defined as daytime resting arterial oxygen tension $<7.5 \mathrm{kPa}$ and/or arterial carbon dioxide tension $>8.0 \mathrm{kPa}$ on air), uncontrolled heart failure, arrhythmia, moderate or severe bulbar weakness or inspiratory positive airway pressure (IPAP) on their current ventilator $>25 \mathrm{cmH}_{2} \mathrm{O}(2.44 \mathrm{kPa})$, or if their usual mask was incompatible with the autotitrating ventilator being tested and they were unable (or unwilling) to swap to a compatible mask. The study was approved by the Royal Brompton and Harefield National Health Service Trust ethics committee (London, UK), and all patients gave informed, written consent.

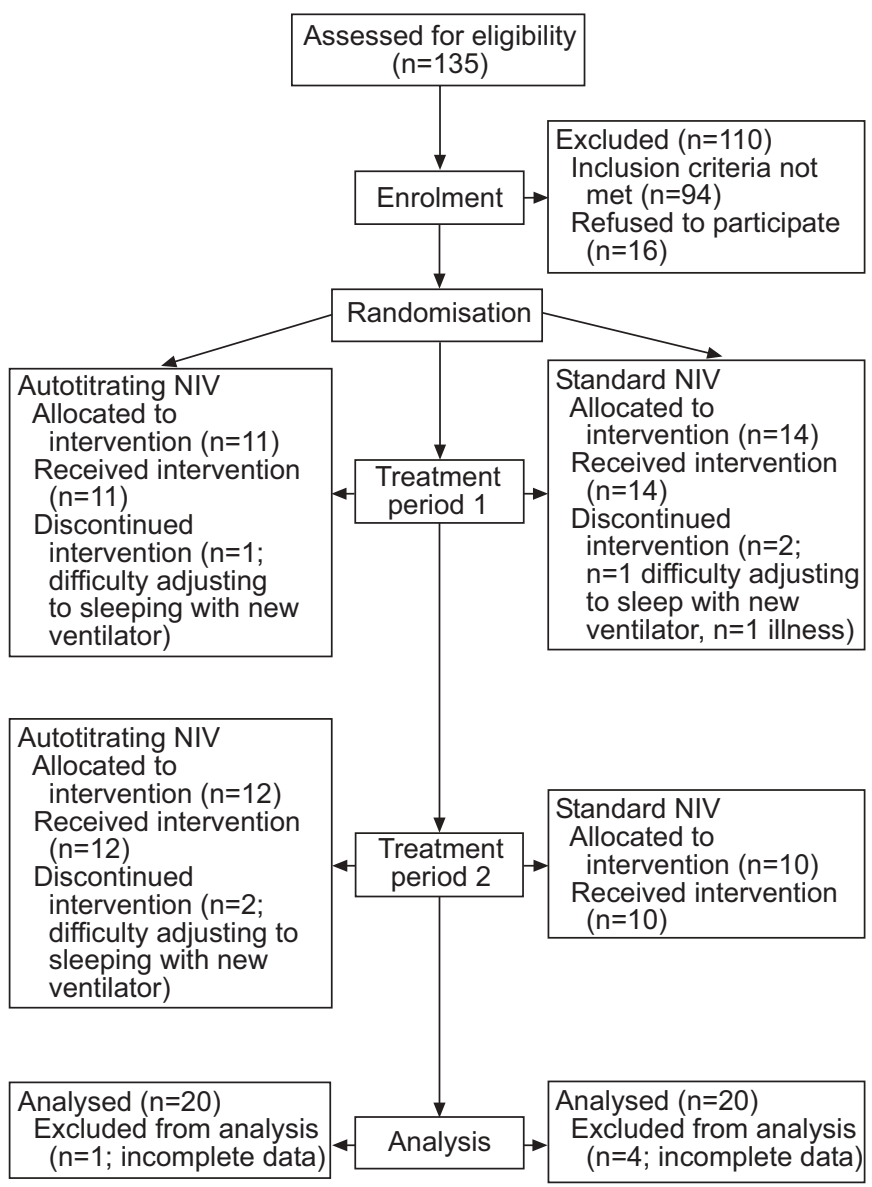

FIGURE 1. Recruitment and flow of patients through each treatment period. NIV: noninvasive ventilation.
The autotitrating NIV had a 1-h learn period, from which it calculated target gross alveolar ventilation or nonanatomical deadspace ventilation (henceforth termed "target alveolar ventilation" for brevity) and variable back-up respiratory rate (RR). During therapy, PSV (IPAP minus expiratory positive airway pressure (EPAP)) was varied between pre-set minimum and maximum limits to achieve the calculated target alveolar ventilation. It responded rapidly when ventilation was well below the target and more slowly when closer to the target. The back-up RR had an optimum level when patient effort consistently failed to trigger the ventilator and a much lower level when the patient was triggering the ventilator. This ensured minimum interference with the patient's spontaneous ventilation and facilitated sleep onset. A more detailed description can be found in the online supplementary material.

\section{Protocol}

The trial design was an interventional, randomised, crossover study comparing NIV administered via an automatically titrated ventilator (AutoVPAP ${ }_{\mathbb{R}}$; ResMed) to that administered via a conventional, expert set-up VPAP III (VPAP III ST-A; ResMed). Both ventilators were used for 1 month in random order, and patients used the ventilator each night.

Patients attended hospital for a baseline assessment, which included measurement of arterialised capillary blood gas tensions, $\mathrm{Ptc}_{\mathrm{tc}} \mathrm{CO}_{2}$, arterial oxygen saturation $\left(\mathrm{Sa}, \mathrm{O}_{2}\right)$, spirometry, noninvasive respiratory muscle strength and arm span. Following these measurements, patients were randomised to either autotitrating or standard NIV. Details of the randomisation process are available in the online supplementary material.

At the end of the first treatment period all baseline measurements were repeated with the exception of arterial blood gases (fig. 2). Subjective tolerance of the ventilator was assessed using visual analogue scales (VAS). Patients underwent overnight polysomnography, including $P_{\mathrm{tc},} \mathrm{CO}_{2}$ measurement, and 24-h Holter monitoring, which were carried out in the sleep clinic or in the patient's home, depending on patient preference. If the patient elected to have polysomnography at home, both studies were conducted in the home, and similarly with patients having in-laboratory studies. Following the first sleep study, the patient was set up on the second ventilator and began the second 1-month treatment period. At the end of the second treatment period all measurements were repeated (fig. 2).

\section{Ventilator settings}

NIV set-ups were performed by a single, trained technician following a standard set-up procedure.

For the standard NIV treatment period, the ventilator was used in spontaneous/timed mode with IPAP pressure, EPAP and backup RR matched as closely as possible to those of the patient's usual ventilator. These settings had been established by clinical adjustment, to reduce respiratory effort and maximise comfort at the bedside, with subsequent overnight monitoring of $P_{\mathrm{tc},} \mathrm{CO}_{2}$ and $\mathrm{Sa}_{1} \mathrm{O}_{2}$ to ensure optimal nocturnal blood gas control. For patients whose usual ventilator had IPAP but no EPAP, the EPAP on the standard NIV was set to the minimum setting available on both ventilators $\left(4 \mathrm{cmH}_{2} \mathrm{O}(0.39 \mathrm{kPa})\right)$. Appropriate mask type was selected, IPAP minimum (minimum inspiratory time) was set to $1.0 \mathrm{~s}$ and all other values were initially set to defaults. 


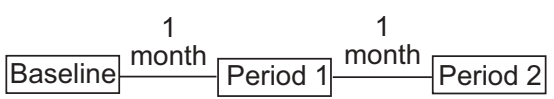

Pulmonary function $\quad \mathrm{X}$ Respiratory $\mathrm{X}$ muscle strength

Subjective tolerance VAS Overnight $\mathrm{Ptc}_{\mathrm{tc}} \mathrm{CO}_{2}$ Polysomnography Holter monitor Arterialised blood gas

$x$
$x$
$x$

$\begin{array}{ll}X & X \\ X & X \\ X & X \\ X & X \\ X & X \\ X & X\end{array}$

FIGURE 2. Timeline detailing tests performed at baseline and end of each treatment period. VAS: visual analogue scales; $\mathrm{Ptc}_{\mathrm{tc}} \mathrm{CO}_{2}$ : transcutaneous carbon dioxide tension.

For the autotitrating treatment period, EPAP was manually set as described for standard NIV, and minimum and maximum PSV values were initially set to maximise the ability of the

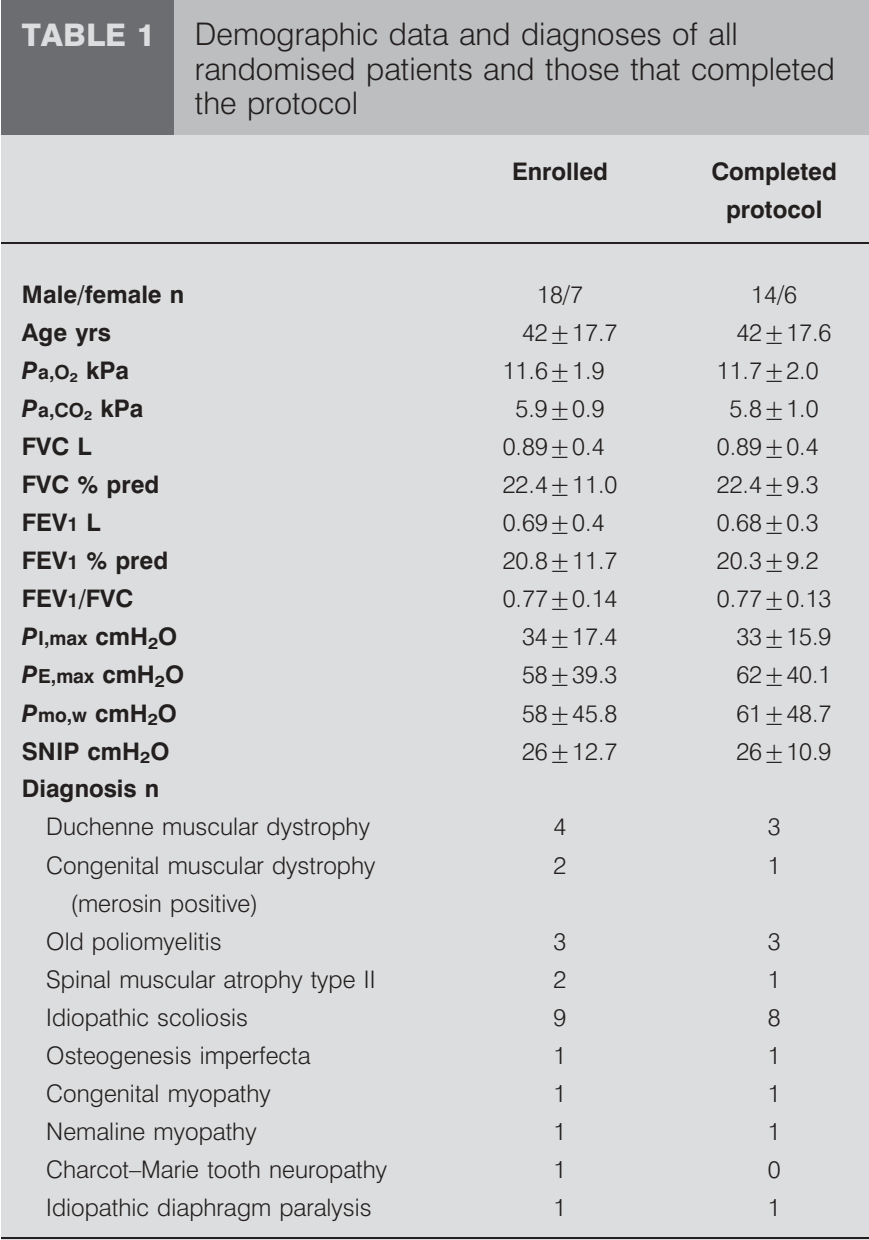

Data are expressed as mean $\pm \mathrm{SD}$, unless otherwise stated. $\mathrm{Pa}_{\mathrm{a}} \mathrm{O}_{2}$ : arterial oxygen tension; $\mathrm{Pa}_{2} \mathrm{CO}_{2}$ : arterial carbon dioxide tension; $\mathrm{FVC}$ : forced vital capacity; \% pred: \% predicted; FEV1: forced expiratory volume in one second; Pl,max: maximal inspiratory pressure at mouth; $P E$,max: maximal expiratory pressure at mouth; $P$ mo,w: maximal whistle mouth pressure; SNIP: sniff nasal inspiratory pressure. $1 \mathrm{cmH}_{2} \mathrm{O}=0.098 \mathrm{kPa}$. ventilator to meet the target alveolar ventilation. Estimated anatomical deadspace was entered using the formula:

$$
V^{\prime} \mathrm{D}=100 \cdot(\mathrm{H} / 1.7)^{3}
$$

where $V^{\prime} \mathrm{D}$ is deadspace ventilation and $\mathrm{H}$ is height (or arm span) in metres. The appropriate mask type was selected and all other values were left at defaults. An initial "learn circuit" function was run to determine the pressure drop across the circuit at different flow rates.

Target alveolar ventilation and back-up RR were based on the patient's breathing over the 1-h learn period, during which the patient sat quietly with their mask on while breathing $4 \mathrm{cmH}_{2} \mathrm{O}(0.39 \mathrm{kPa}) \mathrm{CPAP}$. The patient was asked to remain relaxed but alert and not to doze or talk during this period.

Following set-up on both ventilators, patients had $\geqslant 15 \mathrm{~min}$ of treatment to acclimatise and adjustments were made to settings if a patient was unable to tolerate treatment. Patients could also contact the researcher by telephone if they could not tolerate the settings, and adjustments were made to optimise comfort.

\section{Measurements}

Electroencephalograms (C3A2, C4A1 and O1A2), right and left electro-oculograms, and submental electromyogram (EMG), were recorded using the International 10-20 system of electrode placement [11] and portable polysomnography equipment (Somnoscreen; SOMNOmedics $\mathrm{GmbH}$ and Co., Kist, Germany). Respiration was monitored using mask pressure and abdominal plus thoracic effort bands to record movement. ECG, $\mathrm{Sa}_{2} \mathrm{O}_{2}$, leg EMG and body position were also recorded. Overnight $\mathrm{P}_{\mathrm{tc}, \mathrm{CO}_{2}}$ was measured (TOSCA; Linde Medical Sensors AG, Basel, Switzerland) and daytime $P_{\mathrm{tc},} \mathrm{CO}_{2}$ and resting $\mathrm{Sa}_{2} \mathrm{O}_{2}$ were also measured using the combined earlobe sensor.

The sleep architecture and arousal frequency were analysed using standard criteria $[12,13]$ by a single investigator blinded to the intervention and the subject identity. Sleep study data were analysed for the period the patient was in bed with the ventilator delivering treatment. The mean overnight $\mathrm{Ptc}_{\mathrm{tc}} \mathrm{CO}_{2}$ and $\mathrm{Sa}, \mathrm{O}_{2}$ were analysed over the total sleep period.

The primary endpoint on which the power calculation is based is mean overnight $\mathrm{Sa}_{1} \mathrm{O}_{2}$ on each ventilator at the end of each treatment period. Other outcomes measures were overnight mean and maximum $P_{\mathrm{tc},} \mathrm{CO}_{2}$, minimum $\mathrm{Sa}_{2} \mathrm{O}_{2}, \mathrm{Sa}_{2} \mathrm{O}_{2}$ desaturation index, total sleep time, sleep latency, sleep efficiency, percentage of sleep stages, arousals, arousal index and heart rate variability (HRV). In addition, pulmonary function and noninvasive respiratory muscle strength measurements were made at baseline and at the end of each treatment period, and ventilator use, subjective ventilator tolerance and preference were monitored during each treatment period. Detailed descriptions of these measurements can be found in the online supplementary material.

\section{Statistical analysis}

In total, 20 patients were required, in order to have a $90 \%$ chance of detecting as significant a $2.5 \%$ difference in mean overnight $\mathrm{Sa}, \mathrm{O}_{2}$, with an assumed standard deviation of $3.0 \%(\mathrm{p}=0.05)$. 
TABLE 2 Settings for patient's usual noninvasive ventilation (NIV), standard NIV and autotitrating NIV

\begin{tabular}{|c|c|c|c|c|c|c|c|c|c|c|c|}
\hline \multirow[t]{2}{*}{ ID } & \multicolumn{3}{|c|}{ Usual ventilator settings } & \multicolumn{3}{|c|}{ Standard NIV } & \multicolumn{5}{|c|}{ Autotitrating NIV } \\
\hline & $\begin{array}{l}\text { EPAP } \\
\mathrm{cmH}_{2} \mathrm{O}\end{array}$ & $\begin{array}{c}\text { IPAP } \\
\mathrm{cmH}_{2} \mathrm{O}\end{array}$ & $\begin{array}{c}\text { RR } \\
\text { breaths } \cdot \min ^{-1}\end{array}$ & $\begin{array}{l}\text { EPAP } \\
\mathrm{cmH}_{2} \mathrm{O}\end{array}$ & $\begin{array}{l}\text { IPAP } \\
\mathrm{cmH}_{2} \mathrm{O}\end{array}$ & $\begin{array}{c}\quad \mathrm{RR} \\
\text { breaths } \mathrm{min}^{-1}\end{array}$ & $\begin{array}{l}\text { EPAP } \\
\mathrm{cmH}_{2} \mathrm{O}\end{array}$ & $\begin{array}{c}\text { Minimum } \\
\text { PSV } \\
\mathrm{cmH}_{2} \mathrm{O}\end{array}$ & $\begin{array}{c}\text { Maximum } \\
\text { PSV } \\
\mathrm{cmH}_{2} \mathrm{O}\end{array}$ & $\begin{array}{l}\text { Target alveolar } \\
\text { ventilation } \\
\mathrm{L} \cdot \mathrm{min}^{-1}\end{array}$ & $\begin{array}{c}\text { RR } \\
\text { breaths } \cdot \min ^{-1}\end{array}$ \\
\hline 1 & 6.5 & 25 & 19 & 6.5 & 25 & 19 & 6.4 & 3 & 18.6 & 2.60 & 30 \\
\hline 7 & 7 & 27 & 19 & 7 & 27 & 19 & 7 & 3 & 18 & 4.25 & 25 \\
\hline 10 & NA & 24 & 15 & 4 & 24 & 20 & 4 & 3 & 18 & 4.42 & 25 \\
\hline 11 & 7 & 25 & 14 & 7 & 25 & 14 & 7 & 3 & 18 & 2.89 & 20 \\
\hline 12 & NA & 26 & 16 & 4 & 12 & 15 & 4 & 3 & 21 & 4.93 & 22 \\
\hline 13 & NA & 18 & 17 & 4 & 18 & 17 & 4 & 3 & 18 & 2.80 & 30 \\
\hline 14 & NA & 25 & 15 & 4 & 25 & 15 & 4 & 3 & 18 & 3.06 & 25 \\
\hline 21 & NA & 21 & 15 & 4 & 21 & 15 & 4 & 3 & 21 & 4.33 & 30 \\
\hline 22 & NA & 20 & 26 & 4 & 20 & 26 & 4 & 3 & 18 & 2.89 & 27 \\
\hline 23 & 4 & 10 & 10 & 4 & 10 & 10 & 4 & 3 & 17 & 1.87 & 20 \\
\hline 24 & NA & 24 & 11 & 4 & 20 & 10 & 4 & 3 & 21 & 3.82 & 25 \\
\hline 25 & NA & 21 & 17 & 4 & 19 & 14 & 4 & 3 & 21 & 4.25 & 22 \\
\hline 27 & NA & 18 & 13 & 4 & 18 & 13 & 4 & 3 & 20 & 3.23 & 19 \\
\hline 28 & NA & 20 & 15 & 4 & 19 & 15 & 4 & 3 & 21 & 2.90 & 26 \\
\hline
\end{tabular}

ID: patient number; EPAP: expiratory positive airway pressure; IPAP: inspiratory positive airway pressure; RR: respiratory rate; PSV: pressure-support ventilation; NA: not applicable. $1 \mathrm{cmH}_{2} \mathrm{O}=0.098 \mathrm{kPa}$.

Data obtained at the end of each of the treatment periods were compared, including sleep stages, blood gas values and subjective ventilator tolerance. Normally distributed data were compared initially using a paired t-test and then a linear mixed model; nonparametric data were compared using the Wilcoxon signed rank test. The effect of period and order of ventilator use was determined using a linear mixed model with patients entered as a random effect. Baseline measurements were compared with data obtained at the end of each treatment period, including pulmonary function tests and noninvasive respiratory muscle strength tests. Normally distributed data were compared using the one way ANOVA; nonparametric data were compared using the Kruskal-Wallis ANOVA on ranks test. Values of $\mathrm{p}<0.05$ were considered significant.

\section{RESULTS}

Overall, 25 patients were recruited between November 2005 and April 2007 and five patients withdrew, two patients within 2 days of starting the study and three patients approximately halfway through the study (fig. 1). Demographics and diagnoses are given in table 1 for the 25 randomised patients; 20 patients completed both treatment periods and were included in the intention-to-treat analysis. The majority of patients had restrictive chest wall disease with lung volumes $<1.0 \mathrm{~L}$ and respiratory muscle strength values approximately half those of the normal population [14].
Prior to enrolment, four patients had EPAP on their usual NIV. Table 2 gives the ventilator settings for each patient's usual NIV and the final values for the standard and autotitrating NIV. One patient had supplemental oxygen entrained for the entire study. Five patients had their settings altered after entry into a treatment arm to facilitate comfort and sleep initiation: four on autotitrating NIV and one on standard NIV. One patient had IPAP altered (standard NIV), four had back-up RR altered, two had target alveolar ventilation altered and one had maximum PSV decreased.

Mean overnight $\mathrm{Sa}_{1} \mathrm{O}_{2}$ and $\mathrm{Ptc}_{\mathrm{tc}} \mathrm{CO}_{2}$ values, sleep stage and HRV information for the two ventilators are given in table 3 . There was no significant difference in the mean or minimum overnight $\mathrm{Sa}_{1} \mathrm{O}_{2}, \mathrm{Sa}_{1} \mathrm{O}_{2}$ desaturation index, arousals, sleep efficiency, sleep latency, total sleep time, HRV or compliance. Individual and group mean overnight $\mathrm{Sa}_{\mathrm{a}} \mathrm{O}_{2}$ data at the end of each treatment period are shown in figure $3 a$. However, the mean and maximum overnight $P_{\mathrm{tc}}, \mathrm{CO}_{2}$ were higher on autotitrating compared with standard NIV (median (interquartile range) 7.2 $(6.7-7.7)$ versus $6.7 \quad(6.1-7.0) \mathrm{kPa} \quad(\mathrm{p}<0.001)$, and mean $\pm \mathrm{SD}$ $8.1 \pm 0.82$ versus $7.3 \pm 0.65$ ( $p<0.001$ ), respectively; fig. $4 \mathrm{a}$ and b). There was less stage 1 sleep as a percentage of total sleep time on autotitrating compared with standard NIV (16 \pm 9 versus $19 \pm 10 \%$; $p=0.005$; fig. $3 b$ ) but no significant difference in other sleep stages. The order of ventilator use did not have a significant effect on the differences observed. 


\begin{tabular}{|c|c|c|c|c|}
\hline Variable & Standard NIV & Autotitrating NIV & Difference $(95 \% \mathrm{Cl})$ & $p$-value \\
\hline Subjects $n$ & 20 & 20 & & \\
\hline Mean overnight $\mathrm{Sa}, \mathrm{O}_{2} \%$ & $97(96-98)^{\#}$ & 97 (95-98) & $-0.04(-0.22-1.16)$ & 0.42 \\
\hline Minimum overnight $\mathrm{Sa}_{\mathrm{a}} \mathrm{O}_{2} \%$ & $86(75.3-91.8)^{\#}$ & $91(88.0-95.0)$ & $-2.0(-11.25-0.75)$ & 0.12 \\
\hline Mean overnight $\mathrm{Ptc}_{\mathrm{tc}} \mathrm{CO}_{2} \mathrm{kPa}$ & $6.7(6.1-7.0)^{\#}$ & $7.2(6.7-7.7)$ & $-0.80(-1.18--0.42)$ & $<0.001^{*}$ \\
\hline Maximum overnight $\mathrm{Ptc}_{\mathrm{t}} \mathrm{CO}_{2} \mathrm{kPa}$ & $7.3 \pm 0.65^{\#}$ & $8.1 \pm 0.82$ & $-0.79(-1.14--0.45)$ & $<0.001^{*}$ \\
\hline HRV HF $\mathrm{ms}^{2}$ & $373 \pm 355$ & $309 \pm 331$ & $64(-140-269)$ & 0.51 \\
\hline HRV LF $\mathrm{ms}^{2}$ & 1198 (639-2593) & $930(505-2152)$ & $168(-307-337)$ & 0.35 \\
\hline HRV VLF" $\mathrm{ms}^{2}$ & $7790 \pm 5025$ & $7217 \pm 5195$ & $574(-1408-2555)$ & 0.55 \\
\hline TST h & $6.13(5.40-6.75)$ & $6.22(5.18-6.47)$ & $-0.02(-0.63-0.45)$ & 0.86 \\
\hline Stage 3 and 4 sleep \% TST & $25(18.0-37.4)$ & $31(22.4-41.7)$ & $-4.65(-9.9--1.4)$ & 0.11 \\
\hline Stage 3 and 4 sleep $h$ & $1.60(1.10-1.92)$ & $1.65(1.18-2.30)$ & $-0.30(-0.72--0.02)$ & 0.08 \\
\hline REM sleep \% TST & $12 \pm 6.4$ & $12 \pm 5.4$ & $-0.65(-3.47-2.18)$ & 0.64 \\
\hline Arousals & $98 \pm 70.3$ & $82 \pm 62.1$ & $16.0(-7.00-39.00)$ & 0.16 \\
\hline Arousal index events $\cdot$ hour $^{-1}$ & $21 \pm 9.4$ & $18 \pm 9.4$ & $3.5(-0.17-7.22)$ & 0.06 \\
\hline 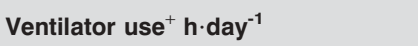 & $7.9(6.76-9.50)$ & $7.8(6.48-9.27)$ & $0.2(-0.16-0.66)$ & 0.16 \\
\hline Ventilator use days & $30 \pm 9.0$ & $28 \pm 5.9$ & $1.5(-1.9-4.9)$ & 0.37 \\
\hline Ventilator use $\%$ days in study & $98(95.7-100.0)$ & $100(89.6-100.0)$ & $0.0(-3.8-1.8)$ & 0.97 \\
\hline
\end{tabular}

Data are expressed as mean $\pm \mathrm{SD}$ or median (interquartile range), unless otherwise stated. $\mathrm{Cl}$ : confidence interval; $\mathrm{Sa}, \mathrm{O}_{2}$ : arterial oxygen saturation; $\mathrm{Ptc}_{\mathrm{tc}} \mathrm{CO}_{2}$ : transcutaneous carbon dioxide tension; HF: high frequency; LF: low frequency; VLF: very low frequency; TST: total sleep time; REM: rapid eye movement. ${ }^{*}$ : $\mathrm{n}=19$; $\because: n=16 ;{ }^{+}$: of days used. *: $p<0.05$, considered significant.

Performance of the ventilators differed as expected: mean \pm SD minute ventilation was significantly lower on autotitrating NIV than standard NIV $\left(5.4 \pm 1.87\right.$ versus $9.0 \pm 3.07 \mathrm{~L} \cdot \mathrm{min}^{-1}$; $\mathrm{p}<0.001)$. The mean \pm SD median mask leak for each patient during autotitrating NIV and standard NIV was $0.07 \pm 0.11$ and $0.28 \pm 0.30 \mathrm{~L} \cdot \mathrm{s}^{-1}(\mathrm{p}<0.001)$, which is within the manufacturer's guidelines for acceptable mask leak and indicates that autotitrating NIV adjusted for leak more effectively.

There was no significant difference in spirometry or respiratory muscle strength tests at baseline and the end of each treatment period (table 4), or between the mean subjective tolerance scores for the two ventilators (table 5). The majority of patients preferred autotitrating NIV compared with standard NIV (fig. 5), though four patients strongly preferred the standard NIV. The mean VAS score for ventilator preference was $0.01 \pm 4.00$. There was no correlation between order of ventilator use and preference.

Two patients developed chest infections: one was admitted to hospital electively for 2 days during the standard NIV treatment period, the other was ill during the autotitrating NIV treatment period but was not admitted to hospital. The number of days on the treatment period during illness was increased to 59 and 61 days, respectively, to allow the patients to recover prior to evaluation of the ventilator.

\section{DISCUSSION}

Automatic titration of NIV using the AutoVPAP produced comparable control of nocturnal oxygenation to standard NIV (VPAP III) that was set clinically, and there were no significant differences in sleep efficiency, total sleep time, sleep latency, arousals, HRV or compliance between set-up modes. There was also no significant impact of either ventilator on pulmonary function and respiratory muscle strength. However, there was a small significant increase in mean overnight $P_{\mathrm{tc}}, \mathrm{CO}_{2}$ on the autotitrating device compared with standard NIV. Increased time spent in deep sleep (stage 3 and 4), significantly reduced percentage of time spent in stage 1 sleep and decreased arousals suggest that the quality of sleep was at least as good on autotitrating NIV as on the expertly set-up standard NIV. Indeed, the reduction in stage 1 sleep might indicate easier sleep initiation and a tendency for a longer period of deep sleep, which in turn may affect nocturnal carbon dioxide level.

A recent study by BATTISTI et al. [15] yielded favourable results when evaluating the efficacy of autotitrating NIV in achieving a clinician-set target alveolar ventilation by automatically varying the PSV within pre-set upper and lower limits. The study by BATTISTI et al. [15] differed from the current study, as the clinician determined the target alveolar ventilation, the variable back-up RR was disenabled, and the patients were stable and conscious with acute respiratory failure in an 

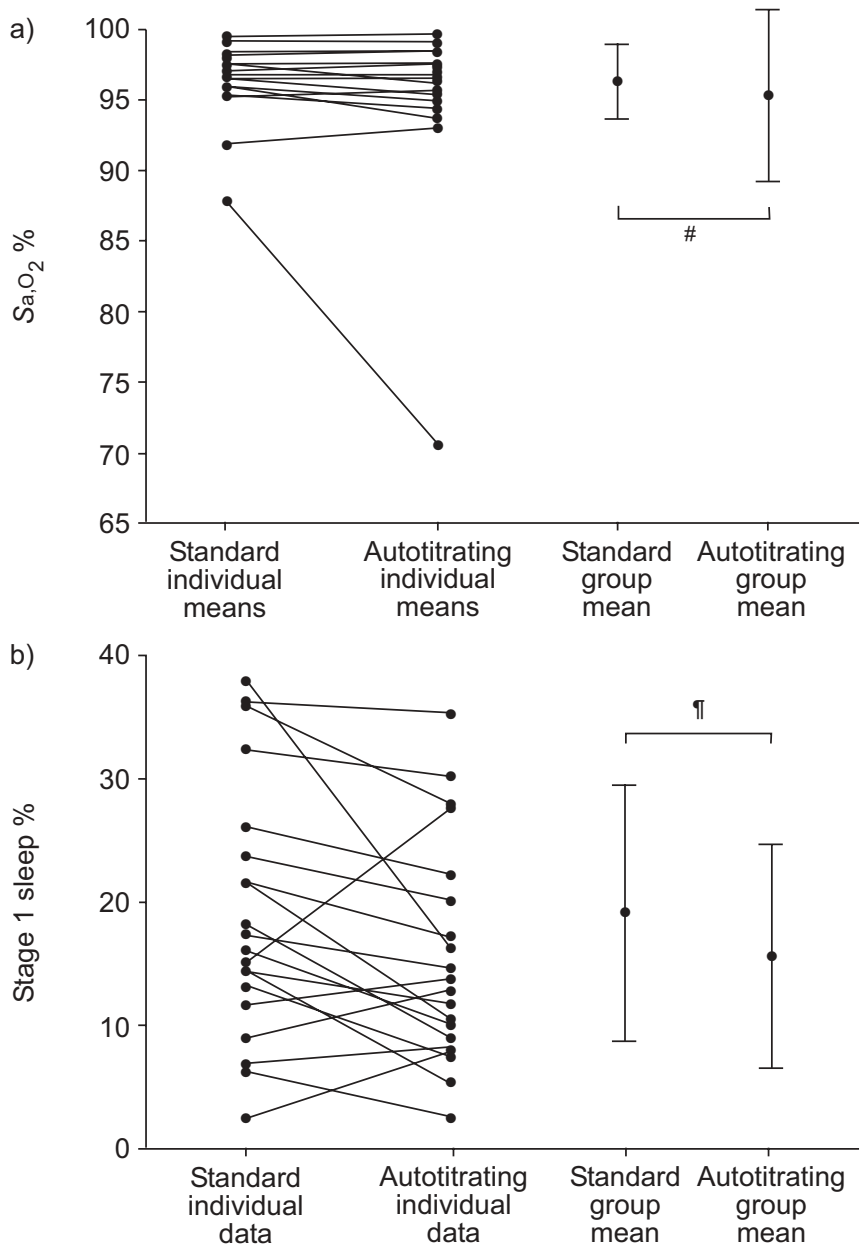

FIGURE 3. a) Mean overnight arterial oxygen saturation $\left(\mathrm{Sa}, \mathrm{O}_{2}\right)$ and b) stage 1 sleep (as percentage of total sleep time while using ventilator) following treatment with standard noninvasive ventilation (NIV) and autotitrating NIV. Individual data and group means are represented. $p<0.05$ was considered significant. ${ }^{*}: p=0.42$ ?: $p=0.005$.
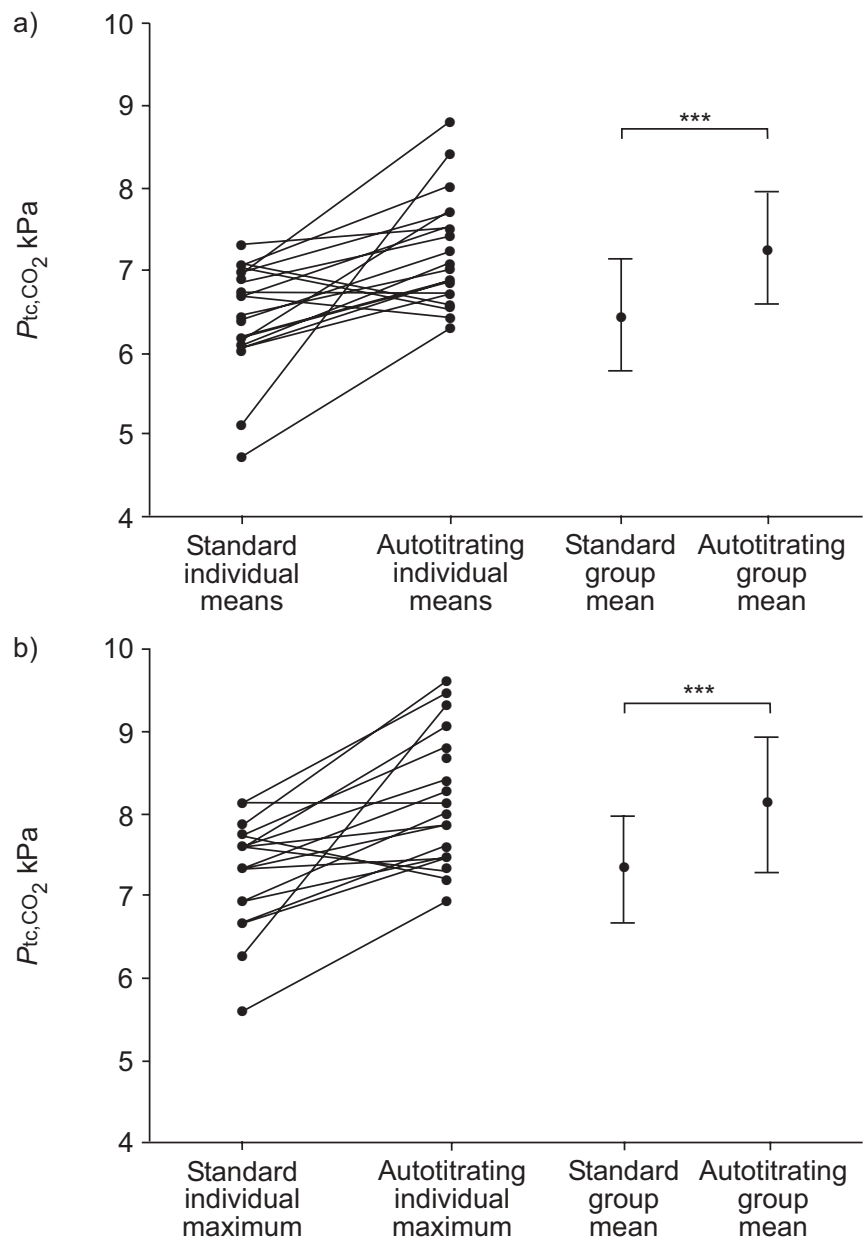

FIGURE 4. a) Mean overnight transcutaneous carbon dioxide tension $\left(\mathrm{Ptc}_{\mathrm{tc}} \mathrm{CO}_{2}\right)$ and b) maximum overnight $P \mathrm{tc}, \mathrm{CO}_{2}$ following treatment with standard noninvasive ventilation (NIV) and autotitrating NIV. Individual data and group means are represented. $p<0.05$ was considered significant. $* * *: p<0.001$

TABLE 4 Pulmonary function and respiratory muscle strength test results following treatment with standard noninvasive ventilation (NIV) and autotitrating NIV compared with baseline

\begin{tabular}{|c|c|c|c|c|c|}
\hline Variable & Baseline & Standard NIV & Autotitrating NIV & Difference $(95 \% \mathrm{Cl})$ & p-value ${ }^{\#}$ \\
\hline Subjects $n$ & 20 & 20 & 20 & & \\
\hline FEV 1 L & $0.66 \pm 0.31$ & $0.62 \pm 0.30^{\circ}$ & $0.62 \pm 0.32^{\circ}$ & $0.002(-0.04-0.04)$ & 0.93 \\
\hline FEV1/FVC & $0.77 \pm 0.13$ & $0.83 \pm 0.19^{\circ}$ & $0.79 \pm 0.14^{\circ}$ & $-0.029(-0.09-0.03)$ & 0.36 \\
\hline $\mathrm{Pl}, \max \mathrm{cmH}_{2} \mathrm{O}$ & $35 \pm 14.5$ & $33 \pm 14.5$ & $35 \pm 15.3$ & $2.133(-0.44-4.71)$ & 0.11 \\
\hline SNIP $\mathrm{cmH}_{2} \mathrm{O}$ & $28 \pm 9.2$ & $29 \pm 11.0$ & $29 \pm 9.2$ & $-0.150(-2.36-2.06)$ & 0.89 \\
\hline
\end{tabular}


TABLE 5 Subjective tolerance data following treatment with standard noninvasive ventilation (NIV) and autotitrating NIV

\begin{tabular}{|c|c|c|c|c|}
\hline Variable & Standard NIV & Autotitrating NIV & Difference $(95 \% \mathrm{Cl})$ & $p$-value \\
\hline Subjects $\mathrm{n}$ & 20 & 20 & & \\
\hline Ease of falling asleep & 84 (67-95) & $86(74-92)$ & $1.0(-5.5-6.0)$ & 0.73 \\
\hline Ease of use of vent & 90 (76-96) & $93(86-97)$ & $-1.5(-8.5-3.5)$ & 0.28 \\
\hline Liked using vent & $75(62-85)$ & $78(53-88)$ & $-1.5(-11.0-16.5)$ & 0.83 \\
\hline
\end{tabular}

Data are expressed as mean \pm SD or median (interquartile range), unless otherwise stated. Visual analogue scale from 0-100 used to score each question, 0 being a negative and 100 being a positive response. A p-value $<0.05$ was considered significant. Cl: confidence interval.

intensive care unit setting. It demonstrated the ability of autotitrating NIV to achieve a given target alveolar ventilation by varying the PSV within pre-set limits, but did not validate the 1-h learn function or variable back-up RR.

HRV analysis was a novel aspect of the current study. Previous studies have shown that the low frequency (LF) band power reflects sympathetic activation, high frequency (HF) band power reflects parasympathetic activation [16], and very low frequency (VLF) band power has been associated with Cheyne-Stokes respiration [17, 18] and OSA [19] in patients with congestive heart failure. The aim in the current study was to identify any alteration in cardiac function in patients using autotitrating compared with standard NIV, and any difference in autonomic activation that could be attributed to altered ventilation, sleep fragmentation, or patient-ventilator synchrony. In fact, LF, HF, VLF and percentage VLF index did not differ significantly on autotitrating compared with standard NIV.

\section{Limitations of the study}

The patient group investigated in the present study had spirometric values approximately one quarter of that predicted for the normal population and respiratory muscle strength approximately half that of the normal population. Despite this reduced pulmonary function and respiratory muscle strength (expected for the patients' conditions), normal daytime blood gas values demonstrated that the patients were adequately ventilated during the day. The ventilators were used to maintain control of nocturnal hypoventilation rather than treat newly diagnosed patients with uncontrolled arterial blood gas tensions. It is possible that patients diagnosed de novo with nocturnal hypoventilation and severe ventilatory failure may be less well served by autotitration of settings compared with those with less severe disease, who may be better suited to

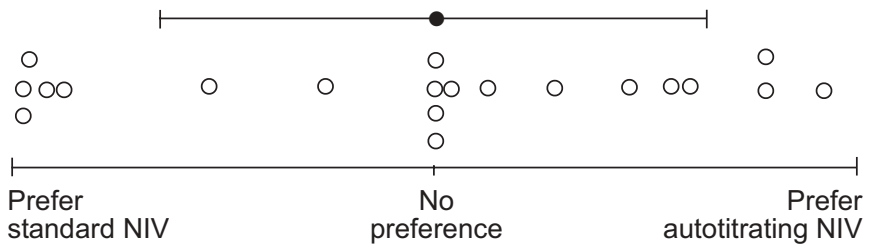

FIGURE 5. A 10-cm visual analogue scale (VAS) was used to assess patient ventilator preference following treatment with standard noninvasive ventilation (NIV) and autotitrating NIV. The VAS centre point indicates no preference for either ventilator. $\bigcirc$ : individual preference; - - - group mean \pm SD. initial set-up on autotitrating NIV as they are likely to have more effective spontaneous ventilation during the 1-h learn period. This remains to be investigated further.

Target alveolar ventilation and back-up RR are automatically titrated, whereas EPAP is manually titrated. If EPAP is suboptimal, obstructive events may reduce NIV efficacy and potentially disturb sleep. In the present study, EPAP for both treatment periods was matched to that of the patient's usual ventilator; however, in clinical use EPAP requires manual titration.

Some patients were initially on single-level positive pressure ventilators without EPAP. During the trial they had EPAP set to the lowest limit $\left(4 \mathrm{cmH}_{2} \mathrm{O}(0.39 \mathrm{kPa})\right)$. However, an EPAP of $5 \mathrm{cmH}_{2} \mathrm{O}(0.49 \mathrm{kPa})$ has been shown to be beneficial in this patient group [20].

The majority of patients opted for home polysomnography rather than laboratory studies. Unattended home polysomnography has been shown to have higher signal failure rates than laboratory studies [21-23]. The current authors had a comparatively low signal failure rate, possibly due to the limited mobility of the present patient group and sensor displacement being lessened by mask and headgear use during the studies. Home polysomnography appears to have no "first night effect" [24], and the evidence suggests that the two scenarios can produce comparable results in respiratory and sleep data $[21,25]$. In the current study, the effect of study location was minimised by limiting patients to the same location for both polysomnographic studies.

In summary, automatically titrated NIV and standard NIV in stable patients with neuromuscular or chest wall disease were equivalent with regard to mean overnight $\mathrm{Sa}_{2} \mathrm{O}_{2}$, and there was no deterioration in respiratory muscle strength or pulmonary function. However, the increased mean overnight $\mathrm{Ptc}_{\mathrm{tc}} \mathrm{CO}_{2}$ indicates that the present patient group was mildly under-ventilated compared with standard NIV, which might, in part, be related to changes in sleep architecture. The current authors also showed that, although leak compensation was superior on autotitrated NIV, mean minute ventilation was significantly reduced. Further adjustments to the algorithm could address this.

Automatic titration of pressure-support ventilation may hold promise for the set-up of noninvasive ventilation in nonacute facilities in which teams are gaining experience in noninvasive ventilation, and also for home set-ups. Further studies should 
examine the efficacy of autotitrating noninvasive ventilation in other patient groups, such as those with newly diagnosed nocturnal hypoventilation who are naïve to noninvasive ventilation.

\section{ACKNOWLEDGEMENTS}

The AutoVPAP and VPAP III devices were provided by ResMed Co. (Bella Vista, Australia). M. Roughton (Research Services, Royal Brompton Hospital, London, UK) provided statistical advice.

\section{REFERENCES}

1 Leger P, Bedicam JM, Cornette A, et al. Nasal intermittent positive pressure ventilation. Long-term follow-up in patients with severe chronic respiratory insufficiency. Chest 1994; 105: 100-105.

2 Simonds AK, Elliott MW. Outcome of domiciliary nasal intermittent positive pressure ventilation in restrictive and obstructive disorders. Thorax 1995; 50: 604-609.

3 Baydur A, Layne E, Aral H, et al. Long term non-invasive ventilation in the community for patients with musculoskeletal disorders: 46 year experience and review. Thorax 2000; 55: 4-11.

4 Bach JR, Alba A, Mosher R, Delaubier A. Intermittent positive pressure ventilation via nasal access in the management of respiratory insufficiency. Chest 1987; 92: 168-170.

5 Kerby GR, Mayer LS, Pingleton SK. Nocturnal positive pressure ventilation via nasal mask. Am Rev Respir Dis 1987; 135: 738-740.

6 Lopez-Campos JL, Garcia Polo C, Leon Jimenez A, Arnedillo A, Gonzalez-Moya E, Fenandez Berni JJ. Staff training influence on non-invasive ventilation outcome for acute hypercapnic respiratory failure. Monaldi Arch Chest Dis 2006; 65: 145-151.

7 Mehta S, Hill NS. Noninvasive ventilation in acute respiratory failure. Respir Care Clin N Am 1996; 2: 267-292.

8 Littner M, Hirshkowitz M, Davila D, et al. Practice parameters for the use of auto-titrating continuous positive airway pressure devices for titrating pressures and treating adult patients with obstructive sleep apnea syndrome. An American Academy of Sleep Medicine report. Sleep 2002; 25: 143-147.

9 d'Ortho MP, Grillier-Lanoir V, Levy P, et al. Constant vs. automatic continuous positive airway pressure therapy: home evaluation. Chest 2000; 118: 1010-1017.

10 Stammnitz A, Jerrentrup A, Penzel T, Peter JH, Vogelmeier C, Becker HF. Automatic CPAP titration with different self-setting devices in patients with obstructive sleep apnoea. Eur Respir J 2004; 24: 273-278.

11 Klem GH, Luders HO, Jasper HH, Elger C. The ten-twenty electrode system of the International Federation. The International Federation of Clinical Neurophysiology. Electroencephalogr Clin Neurophysiol Suppl 1999; 52: 3-6.
12 Rechtschaffen A, Kales A. A Manual of Standardized Terminology, Techniques and Scoring System for Sleep Stages of Human Subjects. Los Angeles, University of California at Los Angeles Brain Information Service/Brain Research Institute, 1968.

13 American Sleep Disorders Association, EEG arousals: scoring rules and examples: a preliminary report from the Sleep Disorders Atlas Task Force of the American Sleep Disorders Association. Sleep 1992; 15: 173-184.

14 Wilson SH, Cooke NT, Edwards RH, Spiro SG. Predicted normal values for maximal respiratory pressures in caucasian adults and children. Thorax 1984; 39: 535-538.

15 Battisti A, Tassaux D, Bassin D, Jolliet P. Automatic adjustment of noninvasive pressure support with a bilevel home ventilator in patients with acute respiratory failure: a feasibility study. Intensive Care Med 2007; 33: 632-638.

16 Malik M, Farrell T, Cripps T, Camm AJ. Heart rate variability in relation to prognosis after myocardial infarction: selection of optimal processing techniques. Eur Heart J 1989; 10: 1060-1074.

17 Ichimaru Y, Yanaga T. Frequency characteristics of heart rate variability produced by Cheyne-Stokes respiration during 24-hour ambulatory electrocardiographic monitoring. Comput Biomed Res 1989; 22: 225-233.

18 Mortara A, Sleight P, Pinna GD, et al. Abnormal awake respiratory patterns are common in chronic heart failure and may prevent evaluation of autonomic tone by measures of heart rate variability. Circulation 1997; 96: 246-252.

19 Roche F, Duverney D, Court-Fortune I, et al. Cardiac interbeat interval increment for the identification of obstructive sleep apnoea. Pacing Clin Electrophysiol 2002; 25: 1192-1199.

20 Elliott MW, Simonds AK. Nocturnal assisted ventilation using bilevel positive airway pressure: the effect of expiratory positive airway pressure. Eur Respir J 1995; 8: 436-440.

21 Fry JM, DiPhillipo MA, Curran K, Goldberg R, Baran AS Full polysomnography in the home. Sleep 1998; 21: 635-642.

22 Portier F, Portmann A, Czernichow P, et al. Evaluation of home versus laboratory polysomnography in the diagnosis of sleep apnoea syndrome. Am J Respir Crit Care Med 2000; 162: 814-818.

23 Redline S, Kapur VK, Sanders MH, et al. Effects of varying approaches for identifying respiratory disturbances on sleep apnoea assessment. Am J Respir Crit Care Med 2000; 162: 369-374.

24 Quan SF, Griswold ME, Iber C, et al. Short term variability of respiration and sleep during unattended non-laboratory polysomnography - The Sleep Heart Health Study. Sleep 2002; 25: 843-849.

25 Iber C, Redline S, Kaplan Gilpin AM, et al. Polysomnography performed in the unattended home versus the attended laboratory setting - Sleep Heart Health Study methodology. Sleep 2004; 27: 536-540. 